\title{
Products Formed by Photosensitized Oxidation of Tocopherols
}

\author{
Ryo YAMAUCHI ${ }^{*}$ and Setsuro Matsushita \\ Research Institute for Food Science, Kyoto University, Kyoto \\ Received May 23, 1979
}

\begin{abstract}
Two isomers of primary products formed by methylene blue sensitized photooxidation of $\delta$-tocopherol were separated by thin layer chromatography. The products were characterized by UV, IR and NMR spectra and were identified to be 8aS- and 8aR-hydroperoxy $\delta$-tocopheryl dienones, respectively. On decomposition of the photooxidized $\alpha$-, $\gamma$ - and $\delta$-tocopherols, tocopheryl quinone and tocopheryl quinone epoxide were formed. The effect of hydroperoxy $\delta$ tocopheryl dienone on methyl linoleate peroxidation was examined. The hydroperoxy dienone formed by the photooxidation of tocopherols did not accelerate lipid peroxidation.
\end{abstract}

Tocopherols as a biological antioxidant have been recognized for their ability to inhibit the free radical chain reaction of lipid peroxidation. ${ }^{1,2)}$ The initiation process of lipid peroxidation is caused by singlet molecular oxygen $\left({ }^{1} \mathrm{O}_{2}\right)^{3 \sim 6)}$ Tocopherols are capable of highly efficient ${ }^{1} \mathrm{O}_{2}$ quenching, both by a physical process and by an irreversible reaction forming products. ${ }^{7 \sim 12)}$ Also one of the functions of tocopherols was proposed to protection of biological lipids from oxidative damages by ${ }^{1} \mathrm{O}_{2}$.

Grams et al. ${ }^{13,14)}$ reported that the products of $\alpha$-tocopherol photooxidation were a mixture of $a$-tocopheryl quinone and quinone epoxide, along with methanol adducts. They suggested that the products were derived from several endoperoxides which resulted from 1,4-cycloaddition across the aromatic ring. On the other hand, Foote et al. ${ }^{15,16)}$ investigated the photooxidation of $\alpha$-tocopherol at low temperature in chloroform and has isolated and characterized hydroperoxy dienone as the primary photoooxidation product. We have reported that photooxidation of $\alpha-\gamma-$ and $\delta$ tocopherols produced two peroxides as primary products and the products after chemical reduction were identified to be tocopheryl hydroquinone. ${ }^{12)}$

This paper deals with the isolation and characterization of the primary products as relatively stable hydroperoxy dienones formed

* Present address: Faculty of Agriculture, Gifu University, Kagamigahara, Gifu. by methylene blue sensitized photooxidation of $\delta$-tocopherol and the identification of decomposed products of photooxidized $\alpha-\gamma-$ and $\delta$-tocopherols. The effect of hydroperoxy dienone on the autoxidation of methyl linoleate were also examined.

\section{MATERIALS AND METHODS}

Materials. $d$ - $\alpha$-tocopherol, $d-\gamma$-tocopherol and $d$ $\delta$-tocopherol were supplied by Eisai Co. The tocopherols were purified by Sephadex LH-20 column chromatography as previously described. ${ }^{12)}$ Methylene blue was purchased from E. Merck. Methyl linoleate purchased from Tokyo Kasei Co. was purified by column chromatography using Florisil ( $100 \sim 200$ mesh). Other reagents were of commercial grade and were used without further purification.

Photooxidation procedure. The method of methylene blue sensitized photooxidation of $\alpha-, \gamma$ - and $\delta$ tocopherols was the same as described in the previous paper. ${ }^{12)}$

Isolation of primary products formed by photooxidation of $\delta$-Tocopherol. $\delta$-Tocopherol $(200 \mathrm{mg})$ and $5 \mathrm{mg}$ of methylene blue were dissolved in $100 \mathrm{ml}$ of ethanol. The solution was irradiated at $20^{\circ} \mathrm{C}$ for $24 \mathrm{hr}$ with a $30 \mathrm{~W}$ tungsten projection lamp. After reaction, the solvent was removed using a vacuum rotary evaporator at below $25^{\circ} \mathrm{C}$. The products were separated by thin layer chromatography (TLC), Merck silica gel $\mathrm{PF}_{254}$ containing $\mathrm{CaSO}_{4}$, using benzene-methanol $(95: 5)$ as the solvent system. Two peroxide fractions of yellow oil were obtained (the yield of more mobile product by the TLC separation was $46 \mathrm{mg}$ and that of less mobile product was $40 \mathrm{mg}$ ). 
Spectroscopy. Ultraviolet (UV) spectra were performed with a Shimadzu UV-200 spectrophometer. Infrared (IR) spectra were taken in a sodium chloride cell with a Hitachi Model 285 infrared spectrophotometer. Nuclear magnetic resonance (NMR) spectra were obtained in $\mathrm{CCl}_{4}$ with a Hitachi-Perkin Elmer Model $90(90 \mathrm{MHz})$. Tetramethylsilane was used as an internal standard and chemical shift of protons was presented as $\delta$ value (tetramethylsilane $=0$ ).

Identification of decomposed products of photooxidized tocopherols. The photooxidation products of tocopherols were separated by the previously described method $^{12)}$ and were left at room temperature for a period of one day to one week. The decomposed products of each photooxidized tocopherol were silylated by hexamethyldisilazane and trimethylchlorosilane in pyridine solution. Gas chromatographymass spectrometry (GC-MS) was carried out with a Shimadzu LKB-9000 apparatus and operational conditions were the same as described previously. ${ }^{12\}}$

Reaction of hydroperoxy $\delta$-tocopheryl dienone with methyl linoleate. Hydroperoxy $\delta$-tocopheryl dienone was dissolved with methyl linoleate (the molar ratio of the compounds was 1:4). The mixture was incubated in the air at $37^{\circ} \mathrm{C}$. Ferric chloride (the molar ratio to the dienone is 0.0002 ) was added to the reaction mixture. At intervals, $5 \mu \mathrm{l}$ of sample were dissolved in $1 \mathrm{ml}$ of ethanol. The sample solution was spotted on a Merck silica gel pre-coated TLC plate $(0.25 \mathrm{~mm}$ thick). After being developed in a solvent system of benzene-methanol $(97: 3)$, the compounds were detected by spraying $50 \% \mathrm{H}_{2} \mathrm{SO}_{4}$ followed by heating at $110^{\circ} \mathrm{C}$ for $10 \mathrm{~min}$. The compounds on the TLC plate were determined from the values densitometrically measured at $350 \mathrm{~nm}$ with zig-zag scanning by a Shimadzu TLC scanner CS-910.

\section{RESULTS}

Primary photooxidation products of $\delta$-tocopherol

The primary photooxidation products of $\alpha$ tocopherol were unstable and readily decomposed on the TLC plate. On the other hand, the primary photooxidation products of $\delta$ tocopherol were relatively stable. The photooxidation products of $\delta$-tocopherol could be separated into two fractions, A and B, by TLC (Fig. 1). UV spectra of compounds A and B showed the same absorption maximum at $230 \mathrm{~nm}$ in ethanol $(\varepsilon=13,000)$. IR spectra of compounds $\mathrm{A}$ and $\mathrm{B}$ are shown in Fig. 2. The spectra showed the presence of a hydro-

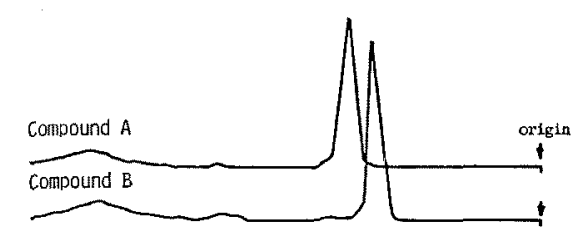

FIG. 1. Densitometric Patterns of the Separated Photooxidation Products of $\delta$-Tocopherol on the TLC Plate.

Development was performed with benzene-methanol (95:5). The optical density of the products on the plate was measured at $230 \mathrm{~nm}$.

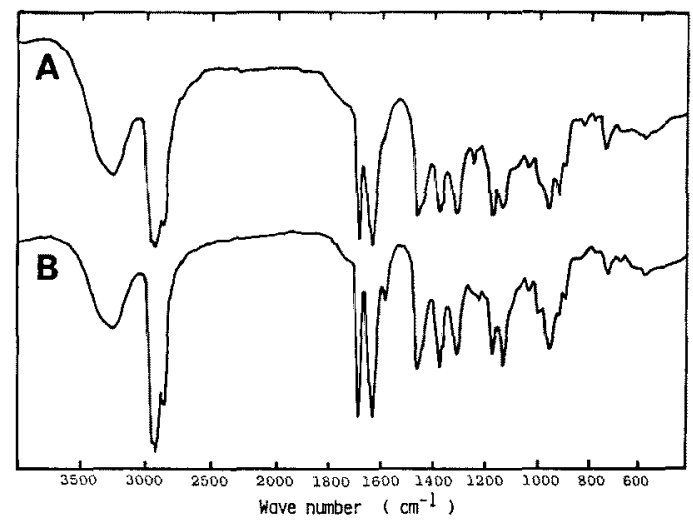

FIG. 2. IR Spectra of Compounds A and B.

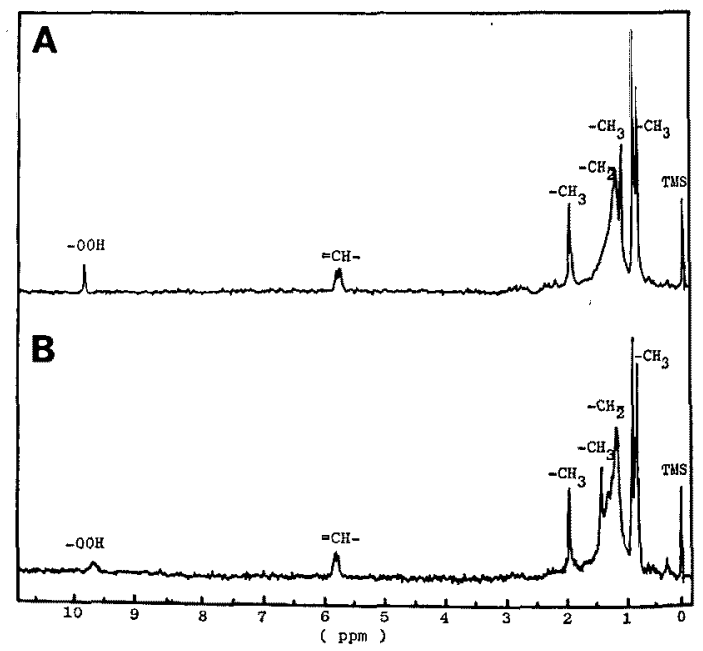

Fig. 3. NMR Spectra of Compounds A and B.

peroxy group $\left(3250 \mathrm{~cm}^{-1}\right)$ and a carbonyl group $\left(1690 \mathrm{~cm}^{-1}\right)$. A remarkable difference between compounds $\mathrm{A}$ and $\mathrm{B}$ was recognized in the range of 1000 to $900 \mathrm{~cm}^{-1}$. Two ab- 
sorption at near 960 and $920 \mathrm{~cm}^{-1}$ appeared in the spectrum of $\mathrm{A}$, while only an absorption at $960 \mathrm{~cm}^{-1}$ was present in that of B. These suggest the presence of cis and trans configuration in the molecule. ${ }^{17}$ NMR spectra of compounds $\mathbf{A}$ and $\mathbf{B}$ are shown in Fig. 3. At down field of $9.97 \mathrm{ppm}$, a sharp signal assigned to hydroperoxy proton was observed (compound $\mathrm{A}$ ), and a broad signal assigned to hydroperoxy proton was observed at $c a .9 .8$ ppm (compound B). After addition of $\mathrm{D}_{2} \mathrm{O}$ to the NMR tube, each peak diminished to the base line. By interpretation of the NMR spectra of compounds $\mathrm{A}$ and $\mathrm{B}$, the geometric relationship could be established. ${ }^{13)}$ When the hydroperoxy group is trans to the 2-methyl group, the 2-methyl group $\left(\delta_{\mathrm{CH}_{3}} 1.11\right)$ is localized in a shielding environment above the plane of the enone system (compound A). When the hydroperoxy group is cis to the 2methyl group, deshielding of the 2-methyl group $\left(\delta_{\mathrm{CH}_{3}} 1.48\right)$ is observed and may be due to 1,3-diaxial interaction of this group with the hydroperoxy group (Compound B). From these spectral data, compound A was identified to be $8 \mathrm{aS}$-hydroperoxy $\delta$-tocopheryl dienone and compound $\mathbf{B}$ was identified to be $8 \mathrm{aR}$ hydroperoxy $\delta$-tocopheryl dienone. ${ }^{16)}$

\section{Decomposed products of photooxidized toco- pherols}

The primary photooxidized products of $\alpha$-, $\gamma$ - and $\delta$-tocopherols were degraded by incubating in the air at room temperature for one day to one week. The decomposed products of each photooxidized tocopherol were silylated and analyzed by GC-MS. Figure 4 shows the gas chromatogram and the mass spectra of the decomposed products of photooxidized $\alpha$-tocopherol. Two main peaks appeared in this figure. From the mass spectrum of each peak, $\alpha$-tocopheryl quinone epoxide trimethylsilyl derivative (peak A); $\mathrm{M}^{+}-\mathrm{CH}_{3}$ $(m / e=519), \quad \mathrm{M}^{+}-\mathrm{C}_{4} \mathrm{H}_{12} \mathrm{SiO} \quad(m / e=430)$, $\mathrm{C}_{21} \mathrm{H}_{45} \mathrm{SiO}^{+}(m / e=341), \mathrm{M}^{+}-\mathrm{C}_{16} \mathrm{H}_{33} \quad(m / e=$ 309 ), and $\alpha$-tocopheryl quinone trimethylsilyl derivative (peak B); $\mathbf{M}^{+}-\mathrm{CH}_{3}(m / e=503)$, $\mathrm{M}^{+}-\mathrm{C}_{4} \mathrm{H}_{12} \mathrm{Si}(m / e=430), \mathrm{C}_{21} \mathrm{H}_{45} \mathrm{SiO}^{+}(m / e=$
341), $\mathrm{M}^{+}-\mathrm{C}_{16} \mathrm{H}_{33}(m / e=293)$, were identified. The gas chromatogram and the mass spectra of decomposed products of photooxidized $\gamma$ and $\delta$-tocopherols are shown in Figs. 5 and 6. From each mass spectrum, $\gamma$-tocopheryl qui-
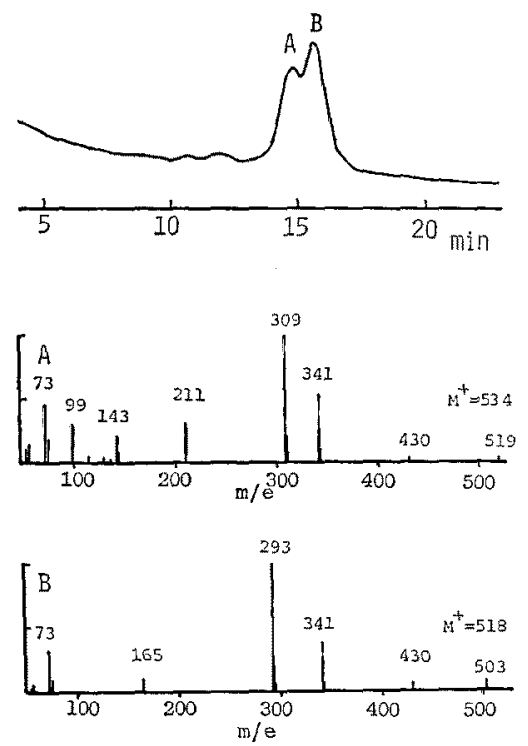

Fig. 4. Gas Chromatogram and Mass Spectra of Decomposed Products of Photooxidized $\alpha$-Tocopherol after Silylation.

For the experiment conditions, see ref. 12.
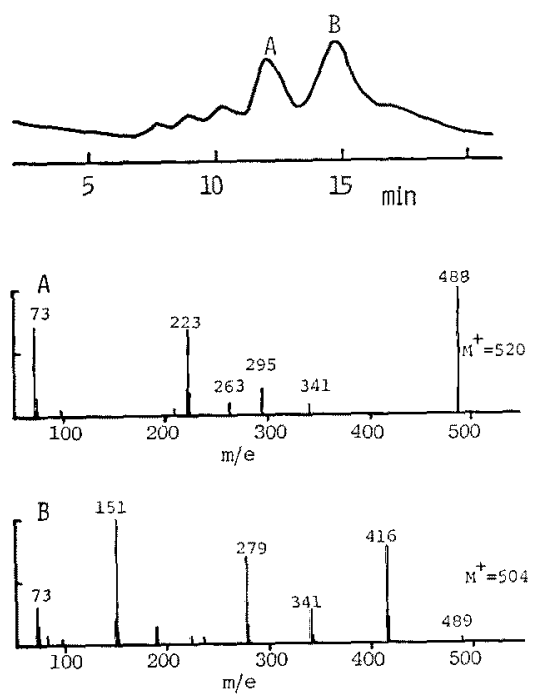

FIG. 5. Gas Chromatogram and Mass Spectra of Decomposed Products of Photooxidized r-Tocopherol after Silylation.

For the experiment conditions, see ref. 12. 

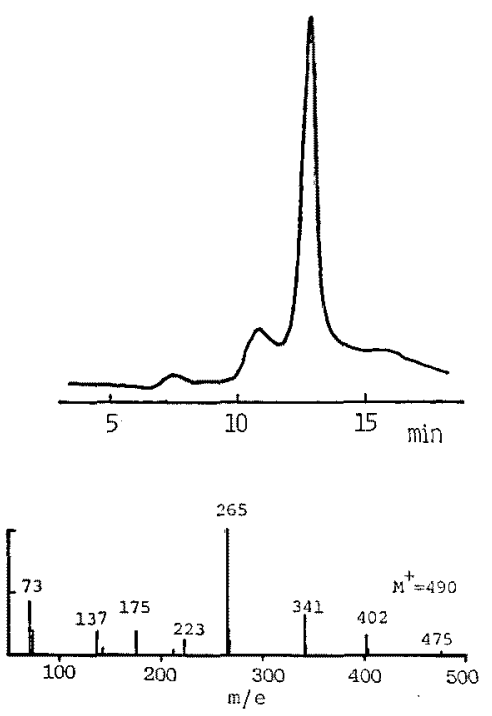

FIG. 6. Gas Chromatogram and Mass Spectrum of a Decomposed Product of Photooxidized $\delta$-Tocopherol after Silylation.

For the experiment conditions, see ref. 12.

none epoxide trimethylsilyl derivative (peak A); $\mathrm{M}^{+}-\mathrm{CH}_{4} \mathrm{O} \quad(m / e=488), \mathrm{C}_{21} \mathrm{H}_{44} \mathrm{SiO}^{+} \quad(m / e=$ 341), $\mathrm{M}^{+}-\mathrm{C}_{16} \mathrm{H}_{33} \quad(m / e=295)$, and $\gamma$-tocopheryl quinone trimethylsilyl derivative (peak B); $\mathrm{M}^{+}-\mathrm{CH}_{3}(m / e=489), \mathrm{M}^{+}-\mathrm{C}_{4} \mathrm{H}_{12} \mathrm{Si}(m / e$ $=416), \mathrm{C}_{21} \mathrm{H}_{44} \mathrm{SiO}^{+}(m / e=341), \mathrm{M}^{+}-\mathrm{C}_{16} \mathrm{H}_{33}$ $(m / e=279)$, were identified (Fig. 5). On the other hand, the decomposed product of photooxidized $\hat{\delta}$-tocopherol gave only one peak on the gas chromatogram and it was identified to be $\delta$-tocopheryl quinone trimethylsilyl derivative from the mass spectrum (Fig. 6); $\mathrm{M}^{+}-$ $\mathrm{CH}_{3} \quad(m / e=475), \quad \mathrm{M}^{+}-\mathrm{C}_{4} \mathrm{H}_{12} \mathrm{Si} \quad(m / e=402)$, $\mathrm{C}_{21} \mathrm{H}_{44} \mathrm{SiO}^{+}(m / e=341), \mathrm{M}^{+}-\mathrm{C}_{10} \mathrm{H}_{33} \quad(m / e=$ 265).

\section{Reaction of hydroperoxy $\delta$-tocopheryl dienone with methyl linoleate}

The compounds containing hydroperoxy group in the molecule may undergo homolytic decomposition to form free radicals, which promote the peroxidation of lipids. Then, the isolated hydroperoxy $\delta$-tocopheryl dienone was incubated with methyl linoleate in the air at $37^{\circ} \mathrm{C}$, and the effect of the hydroperoxy dienone on the lipid peroxidation was investigated. Figure 7 shows the TLC analysis of the com-
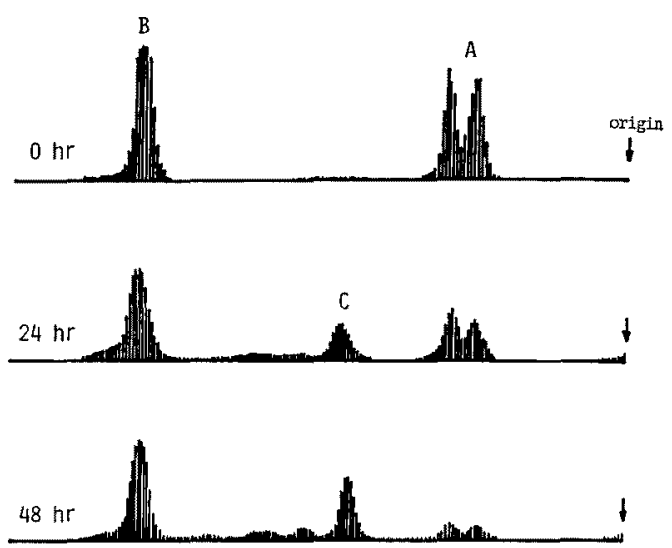

FIG. 7. Densitometric Patterns of Incubation Mixture of Hydroperoxy $\delta$-Tocopheryl Dienone and Methyl Linoleate on the TLC Plate.

The mixture of hydroperoxy $\delta$-tocopheryl dienone and methyl linoleate was incubated at $37^{\circ} \mathrm{C}$ in the air. At 0,24 and $48 \mathrm{hr}$, an aliquot of the mixture was spotted on the TLC plate. After being developed in a solvent system of benzene-methanol $(97: 3)$, the compounds were detected by spraying $50 \% \mathrm{H}_{2} \mathrm{SO}_{4}$ followed by heating at $110^{\circ} \mathrm{C}$ for $10 \mathrm{~min}$. The optical density of the compounds on the plate was measured at $350 \mathrm{~nm}$ by zig-zag scanning by a densitometer. Peak $\mathrm{A}$ is hydroperoxy $\delta$-tocopheryl dienone, peak $\mathrm{B}$ is methyl linoleate and peak $C$ is $\delta$-tocopheryl quinone.

pounds during incubation time. The two peaks of hydroperoxy $\delta$-tocopheryl dienones on the TLC plate diminished with incubation time and the peak of $\delta$-tocopheryl quinone, the decomposed product of the hydroperoxy dienones, was observed. On the contrary, the peak of methyl linoleate on the plate was unchanged. Figure 8 shows the decrease of hydroperoxy $\delta$-tocopheryl dienone and methyl linoleate. The amount of the hydroperoxy dienone decreased with incubation time. But the amount of methyl linoleate in the incubation mixture did not decrease even when ferrous ion was added to the mixture. The only decrease of methyl linoleate was found when ferrous ion was added to the methyl linoleate. In this case, methyl linoleate hydroperoxide, the autoxidation product of methyl linoleate, appeared. The results indicate that the hydroperoxy dienone may prevent the autoxidation of methyl linoleate. 


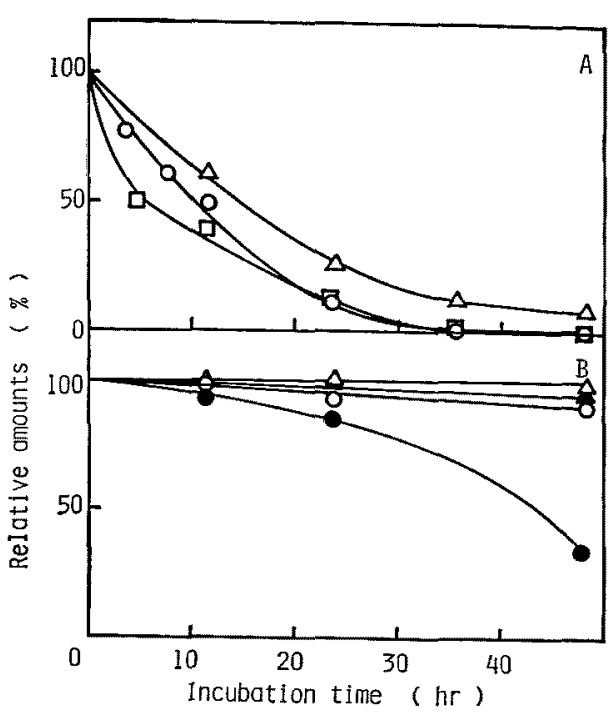

FIG. 8. Changes of Hydroperoxy $\delta$-Tocopheryl Dienone (A) and Methyl Linoleate (B) with Time of Incubation.

The sample was incubated at $37^{\circ} \mathrm{C}$ in the air. (A), $\bigcirc-O$, hydroperoxy dienone; $\Delta-\Delta$, hydroperoxy dienone + methyl linoleate; $\square-\square$, hydroperoxy dienone + methyl linoleate $+\mathrm{FeCl}_{3}$. (B), O-O, methyl linoleate; - - , methyl linoleate $+\mathrm{FeCl}_{3} ; \Delta-\triangle$, methyl linoleate +hydroperoxy dienone; $\mathbf{\Delta}-\mathbf{\Lambda}$, methyl linoleate + hydroperoxy dienone $+\mathrm{FeCl}_{3}$.

\section{DISCUSSION}

It has been proposed that several endoperoxides $^{13,14)}$ or a hydroperoxy dienone ${ }^{15,16)}$ are the primary photooxidation products of $\alpha$ tocopherol. But these products were found to be unstable and could not be isolated. On the other hand, the primary photooxidation products of $\alpha$-tocopherol were relatively stable. The two hydroperoxy dienones were isolated as the primary products formed by photooxidation of $\delta$-tocopherol (Fig. 1). From the spectroscopic data, 8aS-hydroperoxy $\delta$-tocopheryl dienone (2a) and 8aR-hydroperoxy $\delta$ tocopheryl dienone (2b) were identified as shown in Fig. 9. Though Clough et al. ${ }^{16}$ ) predicted these isomeric products from ${ }^{13} \mathrm{C}$ NMR analysis of the photooxidized $\alpha$-tocopherol, they could not separate the compounds. The endoperoxide intermediates proposed by Grams et $a l^{13,14)}$ were not detected in photooxidation. The hydroperoxy dienones were un-<smiles>[R]C1(C)CCc2c(cc(C)c(O)c2C)O1</smiles>

(1)

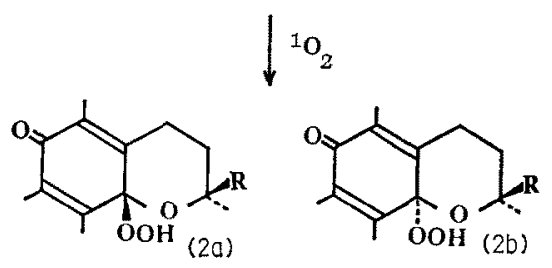

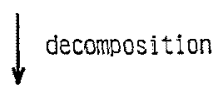<smiles>[R]C(C)(O)CCC1=CC(=O)C(C)=C(C)C1=O</smiles><smiles></smiles>

Fig. 9. Possible Mechanism of Photooxidation of Tocopherols.

stable and decomposed to form tocopheryl quinone (3a) and tocopheryl quinone epoxide (3b), as has previously reported. ${ }^{13 \sim 16)}$ However, the formation ratio of tocopheryl quinone and tocopheryl quinone epoxide was distinct from each photooxidized tocopherol. The decomposed products produced from photooxidized $\alpha$-tocopherol were an equimolar mixture of quinone and quinone epoxide, whereas the decomposed product of photooxidized $\delta$ tocopherol was only $\delta$-tocopheryl quinone (Figs. 4 and 6). The data obtained in this study indicate that the photooxidation of tocopherols (1) in the presence of a sensitizer proceeds by a mechanism as shown in Fig. 9.

In the lipid peroxidation process the breakdown of lipid hydroperoxides gives a chain reaction of autoxidation, ${ }^{18)}$ and consequently the cellular lipids will undergo destructive damages. On the contrary, the hydroperoxy dienone, which was isolated from the photooxidation of $\delta$-tocopherol, did not accelerate the methyl linoleate peroxidation (Fig. 8). Accordingly, it seems likely that the hydroperoxide produced from the photooxidation of tocopherols do not catalyze lipid peroxidation. 


\section{REFERENCES}

1) A. L. Tappel, Ann. N. Y. Acad. Sci., 203, 12 (1972).

2) J. Green, ibid:, 203, 29 (1972).

3) C. S. Foote, Science, 162, 963 (1968).

4) H. R. Rawls and P. J. Van Santen, J. Am. Oil Chem. Soc., 47, 121 (1970).

5) M. M. King, E. K. Lai and P. B. McCay, J. Biol. Chem., 250, 6496 (1975).

6) J. Terao and S. Matsushita, J. Am. Oil Chem. Soc., 54, 234 (1977).

7) G. W. Grams and K. Eskins, Biochemistry, 11, 606 (1972).

8) S.R. Fahrenholtz, F.H. Doleiden, A.M. Trozzolo and A. A. Lamola, Photochem. Photobiol., 20, 505 (1974).

9) C. S. Foote, T.-Y. Ching and G. G. Geller, ibid., 20, 511 (1974).

10) B. Stevens, R. D. Small, Jr. and S. R. Perez, ibid., 20, 515 (1974).

11) D. E. Brabham and J. Lee, J. Phys. Chem., 80,
2292 (1976).

12) R. Yamauchi and S. Matsushita, Agric. Biol. Chem., 41, 1425 (1977).

13) G. W. Grams, K. Eskins and G. E. Inglett, J. Am. Chem. Soc., 94, 866 (1972).

14) G. W. Grams and G. E. Inglett, Lipids, 7, 442 (1972).

15) C. S. Foote, R. L. Clough and B. G. Yee, "Tocopherol, Oxygen and Biomembranes," ed. by C. deDuve and O. Hayaishi, Elsevier NorthHolland Biochemical Press, Amsterdam, 1978, p. 13.

16) R. L. Clough, B. G. Yee and C. S. Foote, J. Am. Chem. Soc., 101, 683 (1979).

17) O.S. Privett and C. Nickell, J. Am. Oil Chem. Soc., 33, 156 (1956).

18) W. O. Lundberg and P. Jarvi, "Progress in the Chemistry of Fats and Other Lipids," ed. by R. T. Holman, Pergamon Press, New York, vol. 9, 1971, p. 377. 